\title{
Timing of Gestational Diabetes Diagnosis by Maternal Obesity Status: Impact on Gestational Weight Gain in a Diverse Population
}

\author{
Teresa A. Hillier, MD, MS, ${ }^{1,2}$ Keith K. Ogasawara, MD, FACOG, ${ }^{3}$ Kathryn L. Pedula, MS, ${ }^{2}$ \\ Kimberly K. Vesco, MD, MPH, FACOG, ${ }^{1}$ Caryn E.S. Oshiro, PhD, RD, ${ }^{2}$ and Jan L. Van Marter, RN, MPA ${ }^{1}$
}

\begin{abstract}
Background: We hypothesized that earlier gestational diabetes mellitus (GDM) diagnosis and treatment of high-risk women would reduce gestational weight gain (GWG) in the first trimester and overall.

Materials and Methods: We evaluated timing of GDM diagnosis among 5,391 pregnant women who delivered singleton births 2010-2013 in a large diverse health maintenance organization (HMO). All GDM screening was by the same oral glucose tolerance testing protocol; GDM treatment protocols were also consistent irrespective of timing of diagnosis. Women without risk factors were universally screened at 24-28 weeks gestation (Usual). Early screening was recommended in obese and other high-risk women at the first prenatal visit; those who screened negative Early were rescreened at 24-28 weeks (Early+Usual).

Results: Average GWG for all women was $12.8 \mathrm{~kg} ; 10.7 \%$ of women were diagnosed with GDM. Average GWG for all women diagnosed with GDM was $10.7 \mathrm{~kg}$, adjusted for gestational age. Women with EarlyGDM averaged $2.4 \mathrm{~kg}$ less GWG than women diagnosed with UsualGDM $(p<0.0001)$. Among obese women, only women diagnosed with EarlyGDM averaged overall GWG within Institute of Medicine (IOM) weight guidelines (mean $8.1 \mathrm{~kg}$ ) and were weight neutral in the first trimester $(-0.2 \mathrm{~kg})$. Overall, $43 \%$ of all pregnant women exceeded IOM GWG guidelines (gained more total weight than recommended); $60 \%$ of obese women exceeded guidelines. Obese women diagnosed with GDM were less likely to exceed IOM guidelines if diagnosed earlier in pregnancy (35\% EarlyGDM vs. 59\% UsualGDM exceeded guidelines, $p<0.0001$ ).

Conclusion: Our results suggest that EarlyGDM diagnosis (and thus treatment) in high-risk women is beneficial for optimizing GWG.
\end{abstract}

Keywords: first trimester screening, gestational diabetes, gestational weight gain

\section{Introduction}

$\mathbf{E}$ ARLY PREGNANCY DIABETES screening, preferably at the initiation of prenatal care, is recommended in high-risk pregnant women (e.g., obesity or prior history of gestational diabetes mellitus $[\mathrm{GDM}])$ to treat unrecognized diabetes in the first trimester before the usual time of GDM screening at 24-28 weeks gestation. ${ }^{1,2}$ GDM is glucose intolerance first recognized in pregnancy that usually resolves after birth, and is associated with adverse maternal and fetal effects. ${ }^{1}$ Lifestyle interventions are the primary therapeutic strategy for women with GDM. ${ }^{3,4}$ After diagnosis, treatment starts with lifestyle management (dietary modifications, physical activity, and weight management depending on pregestational weight), as well as initiating self-monitoring of blood glucose (SMBG) and reporting SMBG results to providers to achieve glycemic goals. ${ }^{1,5}$

Earlier screening is recommended for women at high risk for diabetes in the first trimester (e.g., prior GDM, obesity), ${ }^{1,2}$ to advance diagnosis and treatment. Advancing treatment of hyperglycemia by a trimester with lifestyle management ${ }^{1,5}$ has the added potential to also prevent excessive gestational weight gain (GWG). High-quality evidence indicates that diet or exercise, or both, during pregnancy can reduce the risk

${ }^{1}$ Center for Health Research, Kaiser Permanente Northwest, Portland, Oregon, USA.

${ }^{2}$ Center for Health Research, Kaiser Permanente Hawaii, Honolulu, Hawaii, USA.

${ }^{3}$ Department of Obstetrics and Gynecology, Kaiser Permanente Hawaii, Honolulu, Hawaii, USA.

This study was presented in part at the Pacific Coast Obstetrics \& Gynecology Society meeting, Honolulu, HI, September 2-6, 2015. 
of excessive GWG. ${ }^{6}$ Thus, diagnosing and treating GDM women earlier (rather than waiting until 24-28 weeks gestation) could also potentially avoid excessive GWG.

Excessive GWG is important to prevent: A recent systematic review of $>1$ million pregnant women found that exceeding GWG guidelines was associated with a higher risk of both adverse maternal and infant outcomes. ${ }^{7}$ Moreover, recent data in general populations have identified excessive GWG early in pregnancy as the critical gestational period for risk of high birth weight and other adverse offspring metabolic outcomes. ${ }^{8-12}$ Excessive GWG doubles the risk of macrosomia independent of hyperglycemia. ${ }^{13}$ Thus, the potential to mitigate excess GWG with advancing GDM diagnosis (and thus treatment) by more than a trimester could have high public health impact.

We hypothesized that women diagnosed (and thus treated) earlier with GDM would have less GWG in the first trimester and overall than women diagnosed with UsualGDM, among both obese and nonobese. Universal UsualGDM screening is now recommended for all women at 24-28 weeks gestation. ${ }^{14}$ EarlyGDM screening has also been recommended for high-risk women for some time (e.g., prior GDM, macrosomia, and obesity) by ACOG, ${ }^{15}$ and although part of our clinical practice, was not systematically done for obese women. In 2010, we began a system-wide program to do EarlyGDM screening for all obese pregnant women. This gave us the opportunity to evaluate our hypothesis that earlier GDM diagnosis (with lifestyle management as the primary therapeutic strategy) would result in lower GWG (overall GWG and proportion of women exceeding Institute of Medicine [IOM] guidelines ${ }^{16,17}$ ). As not all high-risk women diagnosed with EarlyGDM were obese, and IOM GWG guidelines differ based on prepregnancy obesity status, ${ }^{16}$ we evaluated our weight gain outcomes separately for the entire screened population of obese and nonobese women.

\section{Materials and Methods}

\section{Research setting}

The study population for our prospective cohort study was drawn from a membership of $>220,000$ in the Kaiser Permanente Hawaii region (KPH). KPH membership is $\sim 20 \%$ of the area's general population and reflects its demographic/ sociographic characteristics. ${ }^{13,18}$ For example, about $10 \%$ of $\mathrm{KPH}$ members are low-income individuals enrolled under the State Health Insurance Plan for Medicaid, a similar proportion of Medicaid members as the state of Hawaii. All members have access to medically necessary services from KPH or by referral from their primary care physician. KPH's Institutional Review Board (IRB) approved this project; it was also approved by the State of Hawaii Department of Health's IRB.

Inclusion criteria for this study were pregnant women without pre-existing diabetes (by ICD-9 coding in the electronic medical record [EMR] ${ }^{19}$ who had GDM screening at KPH during 2010-2013 and delivered live singleton births $(N=5,391$ women $)$.

\section{Gestational diabetes screening: Universal and earlier for high risk}

KPH universally screens all pregnant women for GDM, typically at 24-28 weeks gestation. High-risk women (e.g., prior history of GDM, macrosomia) are generally screened earlier in pregnancy, typically in the first trimester and either treated (if positive) or rescreened again (if negative) at 24-28 weeks gestation. Women with obesity are also considered high risk, but obese women were not systematically screened in the first trimester in our population before 2010 .

Starting in June 2010, KPH implemented a best practice alert (BPA) within the EMR recommending that obstetrics (OB) providers screen all women with obesity in the first trimester of the pregnancy. A notification would "pop up" within the individual patient's EMR that the woman was obese if she met body mass index (BMI) height and weight criteria of BMI $>30 \mathrm{~kg} / \mathrm{m}^{2}$, and that EarlyGDM screening was recommended at the first OB visit. The BPA alert also displayed an order option the provider could easily select to order the first step of a two-step GDM screening protocol (a nonfasting $50 \mathrm{~g}$ glucose challenge test [GCT]; protocol described hereunder). For analytical purposes, we defined Early screening as $<18$ weeks gestation (mean 10.1 and median 10.0 weeks gestation at time of Early screening, respectively). Usual screening was defined as screening $\geq 18$ weeks gestation (mean 28.3 and median 28.0 weeks gestation at time of Usual screening, respectively). High-risk women whose Early screening was negative also had later Usual screening (Early+Usual). Because these were still higher risk women who screened negative in the first trimester (Table 1), we analyzed their weight gain outcomes separately from the women who had universal GDM screening for the first time at 24-28 weeks gestation (Fig. 1).

During the study period (2010-2013), all GDM screening (both Early and Usual) was done by using a two-step screening process with the first step being a nonfasting $50 \mathrm{~g}$, 1-hour GCT, as described by O'Sullivan et al. ${ }^{20}$ Women with GCT $>200 \mathrm{mg} / \mathrm{dL}$ were considered to have GDM and did not undergo further testing. ${ }^{21}$ Otherwise, women with a positive (+) GCT $(\geq 140 \mathrm{mg} / \mathrm{dL})$ received the $100 \mathrm{~g}$, 3-hour oral glucose tolerance test (OGTT) as the second step. GDM diagnosis by OGTT was based on the Carpenter and Coustan $(\mathrm{C} \& \mathrm{C})$ criteria (two or more of the four possible glucose concentrations measured with the $100 \mathrm{~g}$ OGTT are positive: fasting $\geq 95 \mathrm{mg} / \mathrm{dL} ; 1$-hour $\geq 180 \mathrm{mg} / \mathrm{dL} ; 2$-hour $\geq 155 \mathrm{mg} / \mathrm{dL}$; 3-hour $\geq 140 \mathrm{mg} / \mathrm{dL}) .{ }^{15}$ Women who received a positive result on the first screening step (GCT) received the second step (3-hour OGTT) a mean of 7.8 days later (standard deviation $=5.8$ days).

We also had to consider timing of GDM diagnosis as women with normal test(s) for Early screening were routinely rescreened at 24-28 weeks gestation. In some cases, multiple GDM screening tests occurred in each of the early and usual gestational periods. If any of these tests achieved a diagnostic threshold for GDM, then we considered the pregnancy to be positive for GDM at the gestational age women had the positive test.

\section{Gestational diabetes treatment}

The recommended KPH treatment for GDM was the same irrespective of timing of GDM screening and diagnosis. After GDM is diagnosed, lifestyle change (coupled with selfglucose monitoring and feedback) is an essential component of management of GDM and may suffice for the treatment of many women. ${ }^{1,5}$ Specifically, GDM treatment protocols in 
Table 1. Baseline Characteristics by Gestational Diabetes Mellitus Screening Groups

\begin{tabular}{|c|c|c|c|c|}
\hline \multirow[b]{2}{*}{ Characteristic } & \multicolumn{4}{|c|}{ GDM screening group ${ }^{\mathrm{a}}$} \\
\hline & Early only $(\mathrm{n}=280)$ & Early plus Usual ${ }^{\mathrm{b}}(\mathrm{n}=1,124)$ & Usual only $(\mathrm{n}=3,987)$ & $\mathrm{p}^{\mathrm{c}}$ \\
\hline & $\mathrm{n}(\%)$ & $\mathrm{n}(\%)$ & $\mathrm{n}(\%)$ & \\
\hline \multicolumn{5}{|l|}{ Prepregnancy BMI } \\
\hline$<18.5$ & $1(0.4)$ & $13(1.2)$ & $165(4.1)$ & $<0.0001$ \\
\hline $18.5-24.9$ & $35(12.5)$ & $198(17.6)$ & $2,072(52.0)$ & \\
\hline $25.0-29.9$ & $66(23.6)$ & $233(20.7)$ & $1,168(29.3)$ & \\
\hline $30+$ & $178(63.6)$ & $680(60.5)$ & $582(14.6)$ & \\
\hline Maternal age & & & & $<0.0001$ \\
\hline$\leq 25$ & $46(16.4)$ & $252(22.4)$ & $1,200(30.1)$ & \\
\hline $26-30$ & $54(19.3)$ & $316(28.1)$ & $1,173(29.4)$ & \\
\hline $31-35$ & $91(32.5)$ & $308(27.4)$ & $1,079(27.1)$ & \\
\hline $36+$ & $89(31.8)$ & $248(22.1)$ & $535(13.4)$ & \\
\hline \multicolumn{5}{|l|}{ Race } \\
\hline Nonwhite & $264(94.3)$ & $979(87.1)$ & $3,170(79.5)$ & $<0.0001$ \\
\hline White & $14(5.0)$ & $143(12.7)$ & $798(20.2)$ & \\
\hline Unknown $^{\mathrm{d}}$ & $2(0.7)$ & $2(0.2)$ & $19(0.5)$ & \\
\hline \multicolumn{5}{|l|}{ Parity } \\
\hline 0 & $86(30.7)$ & $341(30.3)$ & $1,640(41.1)$ & $<0.0001$ \\
\hline 1 & $77(27.5)$ & $392(34.9)$ & $1,241(31.1)$ & \\
\hline 2 & $60(21.4)$ & $211(18.8)$ & $657(16.5)$ & \\
\hline $3+$ & $57(20.4)$ & $177(15.8)$ & $443(11.1)$ & \\
\hline Unknown $^{\mathrm{d}}$ & 0 & $3(0.3)$ & $6(0.2)$ & \\
\hline \multicolumn{5}{|c|}{ Insulin use (among GDM only) } \\
\hline No. with GDM & 140 & 155 & 282 & \\
\hline Insulin & $82(58.6)$ & $51(32.9)$ & $70(24.8)$ & $<0.0001$ \\
\hline No insulin & $58(41.4)$ & $104(67.1)$ & $212(75.2)$ & \\
\hline \multicolumn{5}{|l|}{ Baby gender } \\
\hline Male & $142(50.7)$ & $568(50.5)$ & $2,044(51.3)$ & 0.9027 \\
\hline Female & $138(49.3)$ & $556(49.5)$ & $1,943(48.7)$ & \\
\hline
\end{tabular}

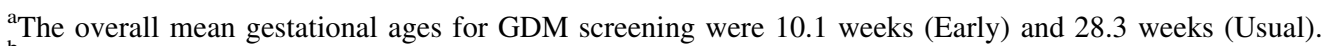

${ }^{\mathrm{b}}$ Some high-risk women had EarlyGDM screening and if screen negative, also had UsualGDM screening later in pregnancy (Early and Usual).

${ }^{\mathrm{c}}$ The $p$-value is based on Mantel-Haenszel test for trend for ordered measures and chi square for categorical measures.

d"Unknown" groups for race and parity were not included in $p$-value calculations.

GDM, gestational diabetes mellitus.

our population include a referral to a dietician for individually tailored lifestyle recommendations, weight management, and self-monitoring of glucose with the following glycemic targets: ${ }^{1,5}$ fasting glucose $<95 \mathrm{mg} / \mathrm{dL}, 1$-hour postprandial $<140 \mathrm{mg} / \mathrm{dL}$, and 2-hour postprandial $<120 \mathrm{mg} / \mathrm{dL}$. If glycemic goals cannot be achieved with lifestyle management, then medication is initiated with insulin the preferred medication of choice ${ }^{5}$ if the patient is willing to use insulin, and the OB provider believes that insulin can be safely administered $^{1}$ (during the study time period, 91\% [203 out of 223] of the patients that received medications for hyperglycemia were treated with insulin; the remaining 20 women were treated with oral hypoglycemic medications).

\section{Centralized laboratory testing and medical records}

All GDM screening laboratories were processed and analyzed by the same methods at KPH regional laboratory, which is certified by the Clinical Laboratory Improvement Amendments (CLIA) and accredited by the College of American Pathologists (CAP) and the American Association of Blood Banks (AABB). The regional laboratory provides comprehensive laboratory analyses to $220,000 \mathrm{KPH}$ members.

KPH maintains administrative and clinical electronic databases on inpatient admissions and deliveries, outpatient visits, laboratory tests, pharmacy dispenses, chronic-disease registries, and outside claims/referrals. All regional databases are linked through each member's unique health record number. Provider diagnoses were used to exclude women with pre-existing diabetes from analyses.

\section{Gestational weight gain}

Prepregnancy and gestational weights were measured and recorded in the EMR (an average of 5.7 measured pregnancy weights for each woman). Measured prepregnancy BMI was defined as the last measurement before estimated date of conception (up to 6 months); if that was not available, we used the earliest measurement during pregnancy (up to 12 weeks gestation). First-trimester GWG is reported only in women with first-trimester measured weights. We did a 


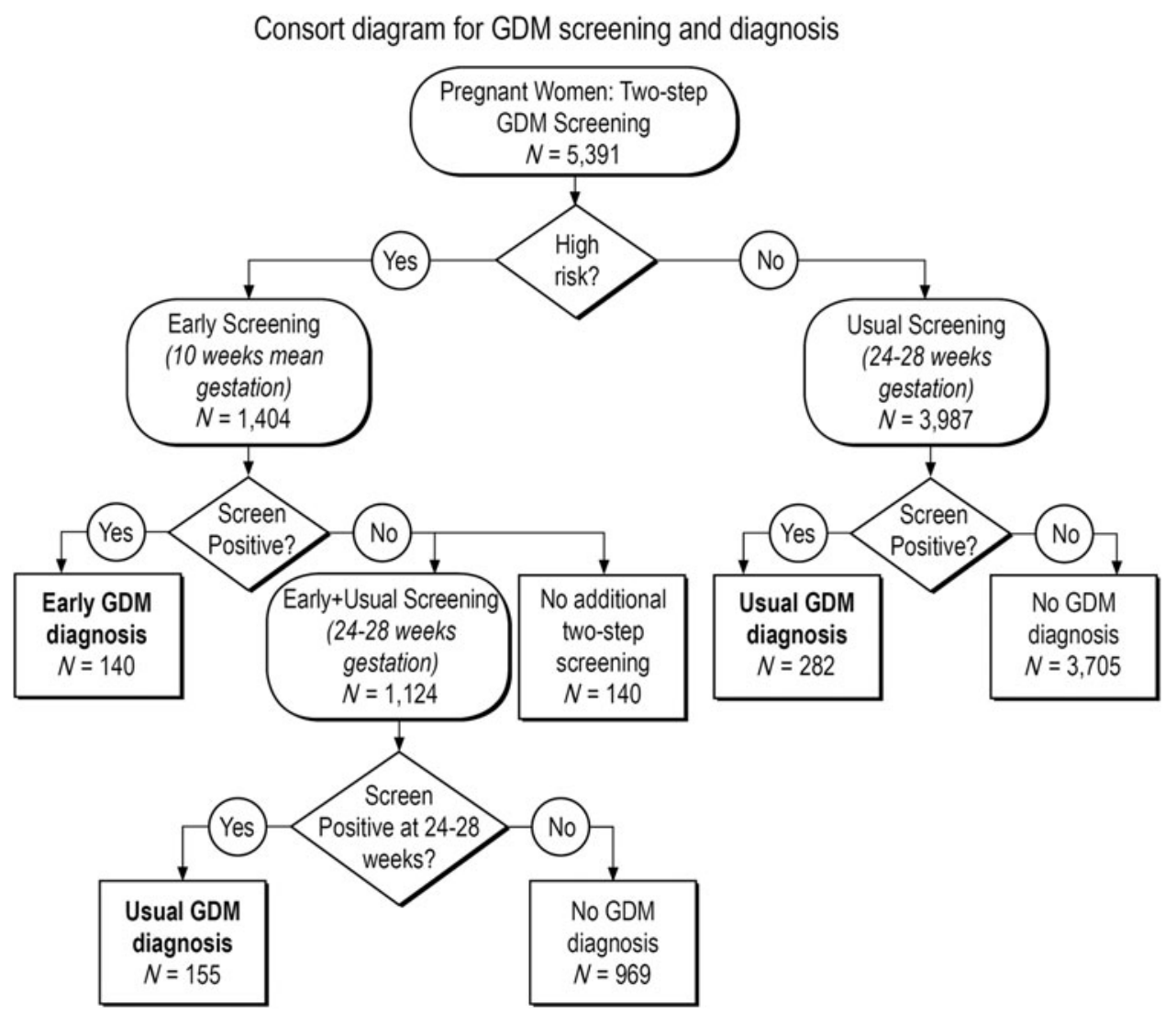

FIG. 1. Consort flow diagram for GDM screening and diagnosis among 5,391 women. High-risk women were screened early (mean 10.1 weeks gestation) and if screen negative in the first trimester, rescreened at 24-28 weeks gestation $(n=1,124)$. The majority of women were universally screened at 24-28 weeks gestation $(n=3,987)$. Overall, $10.7 \%$ women screened were diagnosed with GDM. GDM, gestational diabetes mellitus.

sensitivity analysis between the women with measured prepregnancy weight and women whose first weight measure was after 5 weeks gestation and results were similar.

Excessive weight gain was defined as exceeding IOM pregnancy weight gain guidelines for specific prepregnancy BMI $\left(\mathrm{kg} / \mathrm{m}^{2}\right)$ groups. ${ }^{16}$ IOM guidelines for total weight gain in kilograms are as follows: $12.5-18 \mathrm{~kg}$ for underweight women (BMI <18.5); 11.5-16.0 kg for normal weight women (BMI 18.5-24.9); 7-11.5 kg for overweight women (BMI 25-29.9); 5-9 kg for obese women (BMI $\geq 30$ ). ${ }^{16,17}$ Percentage of women exceeding IOM guidelines was calculated for total GWG. Mean weight gain during the first trimester as well as for the entire pregnancy (adjusted for gestation length) was also calculated.

\section{Classification of race/ethnicity and other covariates}

State birth-certificate records were used for race/ethnicity classification and based on the mother's reported race. ${ }^{18}$ State birth certificates also provided mother's reported parity. Maternal age and measured prepregnancy BMI were obtained from the EMRs. Insulin use was ascertained through the pharmaceutical dispensing databases and was evaluated as overall proportion requiring insulin treatment and its duration.

\section{Statistical analyses}

We conducted all statistical analyses using the SAS Statistical Analysis System version 9.4 (SAS Institute, Cary, NC). Data are complete for all variables except parity $(n=9$ $[0.2 \%])$, and race $(n=23[0.4 \%])$; missing data are listed as "unknown" values in Table 1 and these pregnancies are excluded from group comparisons of these variables. Descriptive statistics, including means, standard deviations, medians, and range, were calculated using the pregnancy as the experimental unit. There were 161 women who delivered more than one singleton pregnancy during the study period. To account for the correlated observations, standard errors were adjusted and statistical comparisons were performed using generalized estimating equations and generalized linear mixed models.

Comparisons involving total GWG are adjusted for gestational age. Other factors considered for adjustment included prepregnancy BMI (both as a continuous variable as well a four-level categorical variable [Table 1]) and insulin use, and the interactions between these variables. The dichotomous obesity measure (BMI $\geq 30$ ) was more strongly associated with GWG than either of the other prepregnancy BMI measures; insulin use was not independently associated with maternal weight gain. In a multivariate model, we found that obesity, GDM, and screening group as well as their interactions were related to maternal weight gain. Because of these significant interactions, we present the stratifications to illuminate the differences among pregnancies within these combinations of factors.

\section{Results}

\section{Population characteristics}

Among 5,391 pregnant women screened for GDM, 1,404 women had Early screening (high risk) and 5,111 women had Usual Screening by universal protocol at 24-28 weeks 
gestation $(3,987+1,124 ;$ Fig. 1$)$. There were 1,124 women who were screened in both time periods as they had negative Early screening and were rescreened at 24-28 weeks [Early+Usual screening]). Early screening for GDM occurred more than one trimester earlier than Usual screening (mean 10.1 weeks gestation vs. 28.3 weeks gestation).

Characteristics of the study population, stratified by screening group, are presented in Table 1. There were 1,440 women $(27 \%)$ in the population who were obese before pregnancy. As Early screening was high risk based, these screening groups (Early and Early+Usual) were more likely to be obese, older, nonwhite, and multiparous. The majority of women diagnosed with EarlyGDM required insulin treatment $(59 \%$ of women with EarlyGDM required insulin treatment vs. $25 \%$ of UsualGDM, $p<0.0001$; Table 1).

\section{GDM prevalence and GWG}

In the overall population, $10.7 \%$ of women were diagnosed with GDM: $2.6 \%$ with EarlyGDM and $8.1 \%$ with UsualGDM. There were more GDM cases overall in the UsualGDM group because the majority of women had their first GDM screening at 24-28 weeks gestation with universal screening as they were not high risk. The proportion of women diagnosed with early GDM screening was higher than usual screening as they were high risk.

Among all women, overall GWG was $12.8 \mathrm{~kg}$ (28 pounds), and $43 \%$ exceeded IOM weight gain guidelines (gained more than recommended for their pregravid BMI; see Table 2). Women with obesity gained $2.1 \mathrm{~kg}$ less on average than nonobese women $(11.2 \mathrm{~kg}$ vs. $13.3 \mathrm{~kg}, p<0.0001)$. However, these women with obesity were more likely to exceed the IOM guidelines than the nonobese $(60.1 \%$ vs. $36.7 \%$, respectively, $p<0.0001$; Table 2 ).

\section{EarlyGDM diagnosis (and thus treatment) is associated with less GWG than UsualGDM}

Total weight gain for all women diagnosed with GDM was a mean of $10.7 \mathrm{~kg}$, adjusted for gestational age. Women with EarlyGDM gained on average $2.4 \mathrm{~kg}$ less than women diagnosed with UsualGDM later in pregnancy $(p<0.0001)$. UsualGDM meant a GDM diagnosis (and resulting treatment) occurred later in pregnancy (mean 28.3 weeks gestation) and included high-risk women who screened negative with early screening as well as those that had universal screening at 24-28 weeks gestation for the first time (Fig. 1). Although women with EarlyGDM were much more likely to require insulin treatment than women with UsualGDM (Table 1), insulin treatment (either overall insulin treatment or its duration) did not impact their weight gain outcomes.

Women with EarlyGDM had the lowest average overall GWG ( $p=0.0005$ compared with women with UsualGDM for both obese and nonobese women). Among obese women diagnosed with GDM, women with EarlyGDM had the lowest rate of exceeding IOM total weight gain guidelines $(p=0.0010 ;$ Table 2).

\section{Additional analyses in obese women}

Among obese women diagnosed with GDM, we examined first trimester and total pregnancy weight gain based on the timing of screening and GDM diagnosis (and thus treatment).
Table 2. Comparison of Weight Gain During Pregnancy by Screening Group and Gestational Diabetes Mellitus, Stratified by Obesity Status

\begin{tabular}{|c|c|c|c|}
\hline & \multicolumn{3}{|c|}{ Weight gain during pregnancy } \\
\hline & $\mathrm{N}$ & $\begin{array}{c}\text { Total } \\
\text { weight } \\
\text { gain }(k g)^{\mathrm{a}}\end{array}$ & $\begin{array}{c}\text { Percentage } \\
\text { exceeding } \\
\text { IOM } \\
\text { guidelines }\end{array}$ \\
\hline Obese & 1,440 & 11.2 & 60.1 \\
\hline Screened Early only ${ }^{\mathrm{b}}$ & 178 & 9.3 & 42.1 \\
\hline No GDM Early & 84 & 10.1 & 50.0 \\
\hline GDM Early ${ }^{\mathrm{c}}$ & 94 & 8.1 & 35.1 \\
\hline Screened Both ${ }^{\mathrm{b}}$ & 680 & 11.8 & 64.0 \\
\hline \multicolumn{4}{|c|}{ (Screen negative Early; rescreened Usual) } \\
\hline No GDM & 592 & 12.0 & 65.0 \\
\hline GDM Usual $^{\mathrm{c}}$ & 88 & 10.7 & 56.8 \\
\hline Screened Usual only ${ }^{\mathrm{b}}$ & 582 & 11.0 & 61.2 \\
\hline No GDM Usual & 514 & 11.0 & 61.1 \\
\hline GDM Usual $^{\mathrm{c}}$ & 68 & 11.4 & 61.8 \\
\hline Nonobese & 3,951 & 13.3 & 36.7 \\
\hline Screened Early only ${ }^{\mathrm{d}}$ & 102 & 10.4 & 28.4 \\
\hline No GDM Early & 56 & 11.2 & 33.9 \\
\hline GDM Early & 46 & 8.6 & 21.7 \\
\hline Screened Both ${ }^{\mathrm{d}}$ & 444 & 13.2 & 43.9 \\
\hline \multicolumn{4}{|c|}{ (Screen negative Early; rescreened Usual) } \\
\hline No GDM & 377 & 13.6 & 46.7 \\
\hline GDM Usual $^{\mathrm{e}}$ & 67 & 10.8 & 28.4 \\
\hline Screened Usual only ${ }^{\mathrm{d}}$ & 3,405 & 13.5 & 36.0 \\
\hline No GDM Usual & 3,191 & 13.6 & 36.6 \\
\hline GDM Usual $^{\mathrm{e}}$ & 214 & 11.6 & 26.6 \\
\hline
\end{tabular}

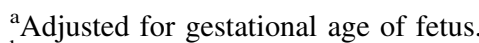

${ }^{\mathrm{b}} p<0.0001$ for total weight gain; $p<0.0001$ for percentage exceeding IOM guidelines comparing three screening groups in obese women.

${ }^{c} p=0.0005$ for total weight gain; $p=0.0010$ for percentage exceeding IOM guidelines comparing GDM among three screening groups in obese women.

${ }^{\mathrm{d}} p<0.0001$ for total weight gain; $p=0.0010$ for percentage exceeding IOM guidelines comparing three screening groups in nonobese women.

${ }^{\mathrm{e}} p=0.0005$ for total weight gain; $p=0.7216$ for percentage exceeding IOM guidelines comparing GDM among three screening groups in nonobese women.

IOM, Institute of Medicine.

Notably, obese women diagnosed with EarlyGDM (and thus treated earlier in pregnancy) were the only group that had an overall average gestational gain that was within IOM recommendations of $<9.0 \mathrm{~kg}$ (mean $8.1 \mathrm{~kg}$ ), and was weight neutral $(-0.2 \mathrm{~kg})$ in the first trimester (Fig. 2). Furthermore, these women diagnosed with EarlyGDM also had the least overall weight gain among the GDM women $(p<0.0001$; Fig. 2).

We then examined the extent to which women with obesity exceeded the IOM guidelines relative to the presence or absence of a GDM diagnosis, as well as timing if diagnosed (EarlyGDM, UsualGDM, and No GDM). EarlyGDMdiagnosed women had a much longer duration of treatment starting in the first trimester on average, compared with UsualGDM (mean diagnosis at 10 weeks vs. 28 weeks gestation, respectively). With that treatment advanced to the first trimester, women with EarlyGDM diagnosis were the least 
Weight Gain for First Trimester and Entire Pregnancy Among Obese Women Diagnosed with GDM

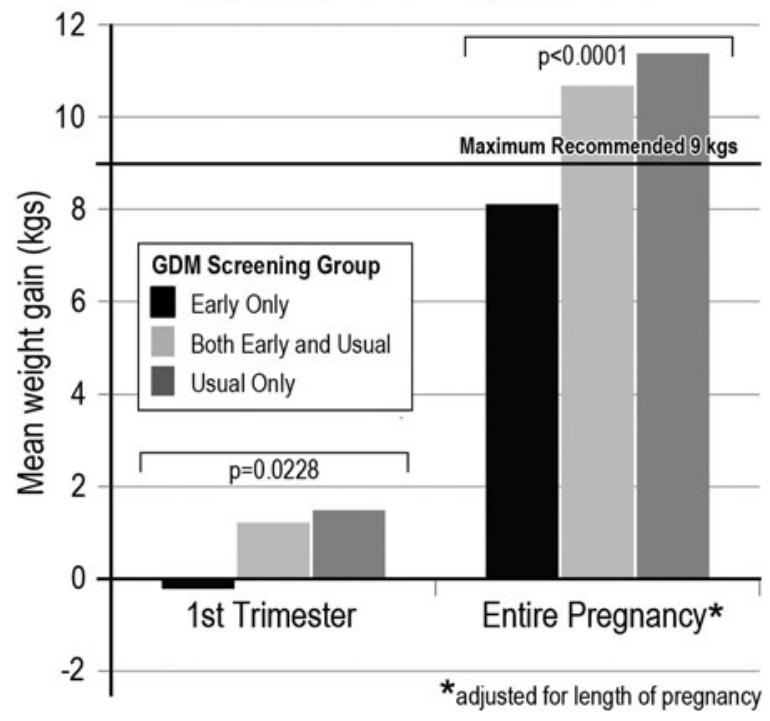

FIG. 2. Average weight gain (in kilograms) among obese women diagnosed with GDM, based on their timing of GDM screening and GDM diagnosis. The three screening groups are: Early Only screened positive and treated early; Early and Usual screened negative early then positive for GDM with usual screening; Usual Only were eligible for early screening as obese but did not have GDM screening and diagnosis until later in pregnancy. Obese women diagnosed with EarlyGDM in the Early Only screening group (and thus received early treatment, including lifestyle management) was the only group that had average weight gain that was within IOM weight gain guidelines of $5-9 \mathrm{~kg}$ (EarlyGDM women gained a mean of $8.1 \mathrm{~kg}$ ) and was weight neutral in the first trimester $(-0.2 \mathrm{~kg})$.

likely group to exceed IOM guidelines ( $p<0.001$; Fig. 3 ). Since exceeding by even a fraction of a kilogram of weight gain results in "exceeding IOM guidelines" we also further stratified exceeding IOM to those who markedly exceeded IOM by $>30 \%$ (an average of $17.1 \mathrm{~kg}$ total weight gain for all women with obesity who exceeded IOM by $>30 \%$ ), and women with EarlyGDM were half as likely to exceed IOM by $>30 \%$ (Fig. 3).

\section{Discussion}

Women diagnosed with EarlyGDM had less GWG in the first trimester and overall than women diagnosed with UsualGDM, among both obese and nonobese subsamples. Moreover, screening positive for GDM in the first trimester of pregnancy (and thus receiving early diagnosis and treatment) was associated with better weight gain outcomes, compared with receiving a negative early screening result, among women at high risk of GDM. Among obese women diagnosed with GDM, only the EarlyGDM group, for whom treatment began in the first trimester, averaged weight gain within IOM weight gain guidelines (mean weight gain $8.1 \mathrm{~kg}$ ). Obese women with EarlyGDM were less likely to markedly exceed IOM guidelines than obese women with UsualGDM or who were never diagnosed with GDM (Fig. 3).
These results are particularly striking as insulin therapy can be associated with weight gain and a much higher percentage of women with EarlyGDM required insulin treatment compared with those with UsualGDM (Table $1, p<0.0001$ ). Thus, even though patients diagnosed with EarlyGDM had more severe cases (higher need for insulin) and insulin treatment could bias toward not finding an effect (with more weight gain), early screening, diagnosis, and thus treatment beginning at a mean of 10 weeks of gestation was associated with better weight gain in these patients than those diagnosed with UsualGDM. These results suggest that among high-risk women, early treatment for GDM improves weight gain, beginning in the first trimester, resulting in better overall weight gain than if they did not receive GDM screening (and treatment) until the usual time of 24-28 weeks gestation.

There is a surprising paucity of data about EarlyGDM screening and diagnosis, and lack of evidence about EarlyGDM in US women is recognized as a high-priority research gap. ${ }^{22,23}$ Bartha et al. found Spanish women diagnosed with GDM early in pregnancy had higher rates of insulin use and worse maternal and perinatal outcomes. ${ }^{24}$ We also have previously reported that EarlyGDM ( $\leq 16$ weeks) appears to be a more severe phenotype, based on a more than doubled risk of needed incident insulin therapy compared with UsualGDM identified at 24-28 weeks gestation (odds ratio $=2.5 ; 95 \%$ confidence interval $2.1-3.1$ ). ${ }^{21} \mathrm{We}$ are not aware of prior studies evaluating the impact of EarlyGDM diagnosis and treatment of high-risk women on GWG in a diverse population.

Recent data have identified excessive GWG in the first trimester as the most important critical window to prevent adverse outcomes. ${ }^{8-12}$ Retnakaran et al. measured pregravid and 10 intervals of weight change in pregnancy among a cohort of >1,000 women: only weight gain before 18 weeks was associated with infant birth weight, after adjusting for maternal prepregnancy BMI and other factors. ${ }^{8}$ Others have found that excessive early GWG (before 18-20 weeks) increases risk of large-for-gestational age babies, ${ }^{9}$ is associated with higher cord blood insulin and C-peptide, ${ }^{10}$ and increased offspring obesity, waist circumference, and adverse cardiovascular risk factors up to age $9 .{ }^{11,12}$

Lifestyle interventions provide benefits to women with GDM and their babies, and are useful as the primary therapeutic strategy. ${ }^{3}$ As before 18 weeks gestation appears to be a critical prevention window, our results that women with EarlyGDM diagnosis had the best weight gain outcomes overall and in the first trimester support that Early screening of high-risk women to advance GDM diagnosis and treatment may be an important prevention strategy to improve GDMassociated outcomes in which obesity and GWG are also risk factors. Moreover, as early intervention in the first trimester is increasingly recognized as a prevention opportunity, ${ }^{25}$ our results help fill an important research gap about maternal weight gain outcome benefits of EarlyGDM diagnosis. Although our study was designed to determine how advancing EarlyGDM screening and diagnosis (and thus treatment) affected weight gain compared with UsualGDM in the context of the entire screened population, our finding that high-risk women who screened negative in the first trimester but were later diagnosed with GDM (Early+Usual) had the worst weight gain outcomes is important. Presumably women in the EarlyGDM group had better weight outcomes because of the 
FIG. 3. Women with obesity, stratified by GDM status (EarlyGDM, UsualGDM, and No GDM), and achievement of IOM recommended GWG for women with obesity (IOM guidelines for obese women are $5-9.1 \mathrm{~kg}){ }^{16,17}$ We additionally stratified exceeding IOM guidelines ( $>9 \mathrm{~kg}$, or 20 pounds) into either exceeding IOM by $1 \%-30 \%$, or markedly exceeding IOM guidelines by $>30 \%$ (average weight gain was $17.1 \mathrm{~kg}$ total weight gain for all women with obesity who exceeded IOM by $>30 \%$ ). Obese women diagnosed with EarlyGDM were most likely to gain within IOM weight gain guidelines. In contrast, the majority of obese women diagnosed with UsualGDM later in pregnancy or without GDM exceeded IOM weight gain guidelines, and were nearly twice as likely to exceed guidelines by $>30 \%$.
Achievement of IOM Recommended Gestational Weight Gain Based on Timing of GDM Diagnosis versus No GDM Among Obese Women

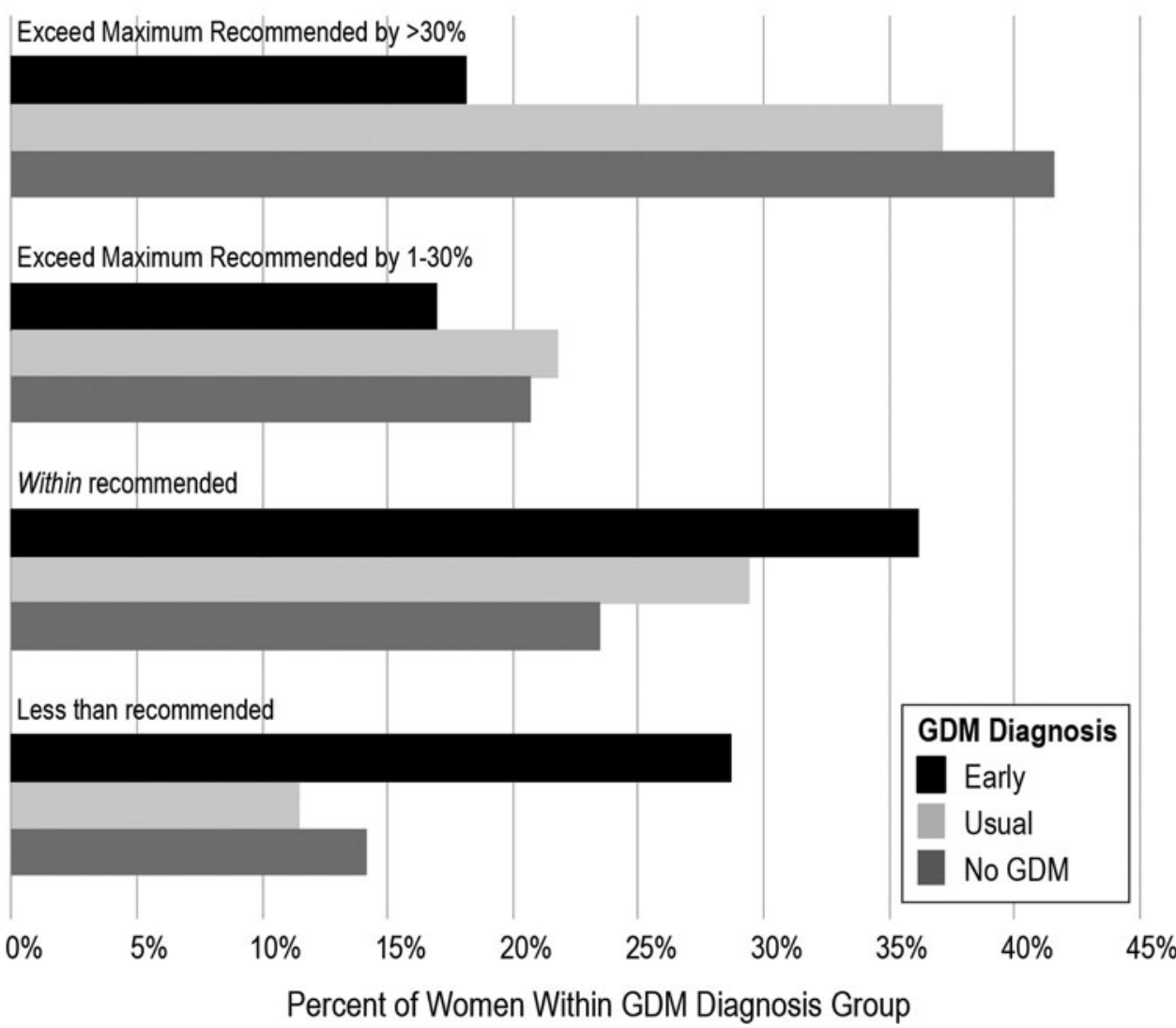

treatment and guidance they received after their early diagnosis. This suggests that a behavioral lifestyle management intervention for high-risk women who did not screen positive for GDM could lead to similar weight outcomes. It is possible, however, that receiving a GDM diagnosis and following an intensive blood glucose monitoring and case management protocol were also essential to weight management in the EarlyGDM group. Future research should evaluate whether a lifestyle management intervention alone could benefit highrisk women who screen negative in the first trimester.

Early pregnancy GDM is a high-priority NIH research gap, as both how to define it is controversial and how early GDM diagnosis affects pregnancy outcomes is unknown. ${ }^{22,23}$ Newly identified diabetes in the first trimester historically has been defined as GDM, and recently also as overt DM by some experts. ${ }^{15}$ The best way to screen for unrecognized diabetes in the first trimester is controversial and lacking data. ${ }^{22,23}$ and some experts recommend screening tests such as $\mathrm{HbA} 1 \mathrm{c}$ with criteria diagnostic for diabetes outside of pregnancy in addition to OGTT screening in the first trimester. ${ }^{1,5}$ Owing to physiological increases in red blood cell turnover, HbA1c levels fall during a normal pregnancy, and thus may be less accurate during pregnancy. ${ }^{5}$ Research to validate HbA1c against pregnancy outcomes is recognized as an important research gap to help determine the utility of $\mathrm{HbA1c}$ as a diagnostic test in early pregnancy. ${ }^{22}$ Our protocol was to define GDM as onset or first recognition of diabetes at any time during pregnancy ${ }^{15}$ by two-step diagnosis (GCT followed by OGTT if GCT+), which is a recommended GDM screening strategy irrespective of gestational age. ${ }^{1}$ Moreover, this has an advantage in our analyses as all women in our population are screened by the same methods during pregnancy, as well as treated the same after GDM diagnosis. This consistent two-step OGTT screening allows comparison of the impact of timing of GDM diagnosis on GWG.

The impact of the EarlyGDM diagnosis for high-risk women within the entire population that had universal screening is paramount to interpret results. We further stratified results in the population for UsualGDM, because UsualGDM comprised high-risk women with negative early screening as well as women with lower risk (Table 1 and Fig. 1). Importantly, the group that had the worst weight gain outcomes (regardless of obesity status) comprised women who had both Early and Usual screening (i.e., high-risk women who screened negative early in pregnancy and later screened positive; see Table 2). Thus, it is likely that high-risk women who screen negative for GDM in the first trimester (who then receive no intervention) would also benefit with more intensive weight management as they remain high risk even though their glucose is normal in the first trimester. Future research should evaluate the impact of a lifestyle intervention (compared with no intervention) on weight gain in high-risk women who screen negative early.

Our study has important strengths. Excessive weight gain in our sample was similar to the US population (64\% of US obese women exceeded IOM guidelines in 2011-2012 $60 \%$ of obese women exceeded in our study). Our population was a large multirace/ethnically diverse of $>5,000$ pregnant 
women with universal maternal GDM screening and followup through birth with multiple measured pregnancy weights in the EMR to evaluate trimester specific and overall weight gain (mean 5.7 of measured weight measures). Our population was also ideal to evaluate the impact of timing of GDM screening and diagnosis on weight gain, as women receive the same GDM treatment protocol at the time of GDM diagnosis regardless of when in pregnancy GDM is diagnosed. Finally, this study also offers the design advantage of evaluating GWG in a "real-world" clinical setting in which the entire broader population is studied, including vulnerable racial/ethnic and socioeconomic sectors that typically do not volunteer to participate in clinical trials, but that are at great risk for GDM.

Every study also has limitations. Although this was a diverse sample of Asians, Pacific Islanders, and Caucasians, it is not representative of the entire US population. However, it does represent important racial groups at high risk for obesity and GDM who are often understudied. Among the 1,440 obese women in our sample, 94 women were diagnosed with EarlyGDM and 68 women who did not receive early screening were diagnosed with UsualGDM. It is unknown what proportion of these 68 women could have been diagnosed with EarlyGDM if they had early screening, but notably their weight gain was worse with the later UsualGDM diagnosis (Table 2). Moreover, only the obese women who screened positive for EarlyGDM (and were thus treated) had an average optimal weight gain within IOM guidelines (Fig. 1). Among 178 obese women with early screening who did not have further testing, 84 were screen-negative, and had lower GWG than women who went on to later Usual screening (Table 2). We presume these early screen-negative women without further testing had some GDM lifestyle management and monitoring although they were heterogenous so we cannot determine why they did better. Finally, there is great controversy with little data on the best screening methods for EarlyGDM, ${ }^{22}$ and it is likely that other methods (such as HbA1c or $75 \mathrm{~g}$ OGTT) would have resulted in a higher overall prevalence of GDM. However, from an analytical standpoint it is ideal that both Early and Usual screening groups were diagnosed with GDM by the same method in our study.

\section{Conclusion}

In summary, we found that early screening of high-risk women to advance diagnosis (and thus treatment) of EarlyGDM was associated with less overall weight gain, and these women with EarlyGDM were also the least likely to exceed IOM weight guidelines. Thus, our results suggest that EarlyGDM screening and diagnosis may be beneficial for tempering GWG by advancing treatment in early pregnancy that entails lifestyle management, including diet and weight management, as first-line treatment for GDM coupled with initiating SMBG and GDM case management. Our finding that EarlyGDM diagnosis and treatment can potentially mitigate excess GWG has high public health impact, and fills an important research gap about diagnosing GDM early in pregnancy. As the majority of obese women exceed IOM weight gain guidelines, our results also suggest that more research is needed on early interventions to support healthy weight gain in all high-risk obese women, especially those who screen negative for an EarlyGDM diagnosis. Future research should investigate the mechanisms by which diagnosis and treatment led to lower weight gain in EarlyGDM patients, and explore whether similar treatment approaches could benefit a broader population of at-risk women.

\section{Acknowledgment}

We thank Ms. Robin Daily for her help in the article preparation.

\section{Author Disclosure Statement}

No competing financial interests exist.

\section{Funding Information}

This study was supported by a grant award 1R01HD058015 from the Eunice Kennedy Shriver National Institute of Child Health and Human Development (to T.A.H.). The funding source had no involvement in the study design; the collection, analysis, or interpretation of data; the writing of the report; or in the decision to submit the article for publication.

\section{References}

1. Committee on Practice Bulletins-Obstetrics. ACOG Practice Bulletin No. 190: Gestational Diabetes Mellitus. Obstet Gynecol 2018;131:e49-e64.

2. American Diabetes Association. 2. Classification and diagnosis of diabetes: Standards of medical care in diabetes2019. Diabetes Care 2019;42:S13-S28.

3. Brown J, Alwan NA, West J, et al. Lifestyle interventions for the treatment of women with gestational diabetes. Cochrane Database Syst Rev 2017;5:Cd011970.

4. Hillier TA, Vesco KK, Pedula KL, Beil T, Whitlock E, Pettitt DJ. Screening for gestational diabetes mellitus: A systematic review for the U.S. Preventive Services Task Force. Ann Intern Med 2008;148:766-775.

5. American Diabetes Association. 14. Management of Diabetes in Pregnancy: Standards of Medical Care in Diabetes2019. Diabetes Care 2019;42:S165-S172.

6. Muktabhant B, Lawrie TA, Lumbiganon P, Laopaiboon M. Diet or exercise, or both, for preventing excessive weight gain in pregnancy. Cochrane Database Syst Rev 2015: Cd007145.

7. Goldstein RF, Abell SK, Ranasinha S, et al. Association of gestational weight gain with maternal and infant outcomes: A systematic review and meta-analysis. JAMA 2017;317: 2207-2225.

8. Retnakaran R, Wen SW, Tan H, et al. Association of timing of weight gain in pregnancy with infant birth weight. JAMA Pediatr 2018;172:136-142.

9. Catov JM, Abatemarco D, Althouse A, Davis EM, Hubel C. Patterns of gestational weight gain related to fetal growth among women with overweight and obesity. Obesity (Silver Spring) 2015;23:1071-1078.

10. Rifas-Shiman SL, Fleisch A, Hivert MF, Mantzoros C, Gillman MW, Oken E. First and second trimester gestational weight gains are most strongly associated with cord blood levels of hormones at delivery important for glycemic control and somatic growth. Metabolism 2017;69: 112-119. 
11. Karachaliou M, Georgiou V, Roumeliotaki T, et al. Association of trimester-specific gestational weight gain with fetal growth, offspring obesity, and cardiometabolic traits in early childhood. Am J Obstet Gynecol 2015;212: 502.e501-514.

12. Fraser A, Tilling K, Macdonald-Wallis C, et al. Association of maternal weight gain in pregnancy with offspring obesity and metabolic and vascular traits in childhood. Circulation 2010;121:2557-2564.

13. Hillier TA, Pedula KL, Vesco KK, et al. Excess gestational weight gain: Modifying fetal macrosomia risk associated with maternal glucose. Obstet Gynecol 2008;112:10071014.

14. US Preventive Services Task Force (USPSTF). Final Update Summary: Gestational Diabetes Mellitus, Screening. 2014. Available at: https://www.uspreventiveservicestask force.org/Page/Document/UpdateSummaryFinal/gestationaldiabetes-mellitus-screening Accessed January 2, 2019.

15. Committee on Practice Bulletins-Obstetrics. Practice Bulletin No. 137: Gestational diabetes mellitus. Obstet Gynecol 2013;122:406-416.

16. Institute of Medicine (IOM) and National Research Council (NRC). Weight gain during pregnancy: Reexamining the guidelines. Washington, DC: The National Academies Press, 2009.

17. American College of Obstetricians and Gynecologists. ACOG Committee opinion no. 548: Weight gain during pregnancy. Obstet Gynecol 2013;121:210-212.

18. Hillier TA, Pedula KL, Schmidt MM, Mullen JA, Charles MA, Pettitt DJ. Childhood obesity and metabolic imprinting: The ongoing effects of maternal hyperglycemia. Diabetes Care 2007;30:2287-2292.

19. World Health Organization. International Classification of Diseases; Manual of the International Statistical Classification of Diseases, Injuries, and Causes of Death. Geneva, Switzerland, 1977.
20. O'Sullivan JB, Gellis SS, Dandrow RV, Tenney BO. The potential diabetic and her treatment in pregnancy. Obstet Gynecol 1966;27:683-689.

21. Hillier TA, Ogasawara KK, Pedula KL, Vesco KK. Markedly different rates of incident insulin treatment based on universal gestational diabetes mellitus screening in a diverse HMO population. Am J Obstet Gynecol 2013;209: 440-449.

22. Wexler DJ, Powe CE, Barbour LA, et al. Research Gaps in Gestational Diabetes Mellitus: Executive Summary of a National Institute of Diabetes and Digestive and Kidney Diseases Workshop. Obstet Gynecol 2018;132:496-505.

23. Bremer AA. Commentary: Research Gaps in Gestational Diabetes Mellitus: Executive Summary of a National Institute of Diabetes and Digestive and Kidney Diseases Workshop. Front Endocrinol 2018;9:627.

24. Bartha JL, Martinez-Del-Fresno P, Comino-Delgado R. Gestational diabetes mellitus diagnosed during early pregnancy [see comment]. Am J Obstet Gynecol 2000;182:346350.

25. Poon LC, David McIntyre H, Hyett JA, Fonseca EBD, Hod $\mathrm{M}$. The first-trimester of pregnancy - a window of opportunity for prediction and prevention of pregnancy complications and future life. Diabetes Res Clin Pract 2018;145: 20-30.

Address correspondence to: Teresa Hillier, MD, MS

Center for Health Research Kaiser Permanente Northwest 3800 N. Interstate Avenue Portland, OR 97227

USA

E-mail: teresa.hillier@kpchr.org 